\title{
Termografia infravermelha e ultrassonografia Doppler na avaliação dos efeitos da insulação escrotal sobre a dinâmica do fluxo sanguíneo escrotal em touros
}

Flávio Antônio Barca Junior ${ }^{[a]}$, Celso Koetz Junior ${ }^{[a]}$, Gabriel Ribas Pereira ${ }^{[b]}$, Silvio Renato Oliveira Menegassib] ${ }^{[b]}$, Fabio Morotti ${ }^{[c]}$, Júlio Otávio Jardim Barcellos ${ }^{[b]}$, Patrícia da Cruz Fávaro[ ${ }^{[b]}$, Lahys Tuigui Diniz ${ }^{[c]}$, Anne Kemmer Souza ${ }^{[c]}$, Marcelo Marcondes Seneda $a^{[c]}$

\footnotetext{
[a] Universidade do Norte do Paraná (UNOPAR), Arapongas, PR, Brasil

[b] Universidade Federal do Rio Grande do Sul (UFRGS), Porto Alegre, RS, Brasil

${ }^{[c]}$ Laboratório de Reprodução Animal, Universidade Estadual de Londrina (UEL), Londrina, PR, Brasil
}

*Autor correspondente

e-mail: marcelo.seneda@gmail.com

\section{Resumo}

O objetivo deste estudo foi avaliar a dinâmica de temperatura escrotal e do fluxo sanguíneo testicular utilizando termografia infravermelha e ultrassonografia Doppler em touros submetidos à insulação escrotal. Touros Braford ( $\mathrm{n}=8$ ), clinicamente saudáveis e com 18 meses de idade, foram aleatoriamente distribuídos em grupo controle (CON, $n=2$, não submetidos à insulação escrotal) e grupos insulados por 72 horas (G 72, n= 2), 96 horas (G 96, n = 2) e 120 horas ( $120, n=2$ ). A termografia infravermelha e a análise ultrassonográfica Doppler velocimétrica (velocidade, índice de pulsatilidade e índice de resistividade) foram realizadas em quatro períodos diferentes: imediatamente após o insulamento escrotal (M0), com 10 minutos (M10), 30 minutos (M30) e 60 minutos após a insulação escrotal (M60). Os dados foram analisados por ANOVA, Teste T (pareado) e correlação de Pearson com nível de significância de 5\%. Em nenhuma das variáveis foram observadas diferenças entre os grupos insulados (G 72, G 96 e G 120, P > 0,05). A temperatura retal $(38,490 \pm 0,40)$ foi maior em relação à superfície escrotal $\left(32,7^{\circ} \mathrm{C} \pm 0,8 ; \mathrm{P}<0,05\right)$. Animais insulados apresentaram maior temperatura escrotal em $\mathrm{M} 0\left(33,0^{\circ} \mathrm{C} \pm 0,69\right)$ em relação aos períodos $\mathrm{M} 10, \mathrm{M} 30$ e $\mathrm{M} 60\left(30,2^{\circ} \mathrm{C} \pm 1,37 ; 31,63{ }^{\circ} \mathrm{C} \pm 1,57\right.$ e 30,65 ${ }^{\circ} \mathrm{C} \pm 1,04$; respectivamente; $\mathrm{P}$ $<0,05)$. Não houve diferença nos índices de pulsatilidade e resistividade após os períodos de insulação escrotal. No entanto, a velocidade do fluxo sanguíneo foi maior em M10 $(17,11 \mathrm{~cm} / \mathrm{s} \pm 4,22)$ em relação aos períodos M0, M30 e M60 (12,52 cm/s $\pm 5,09 ; 14,27$ cm/s $\pm 4,94$ e 14,30 cm/s $\pm 2,91$; respectivamente; $\mathrm{P}<0,05)$. Correlação positiva $(93,1 \%)$ foi encontrada entre os índices pulsatilidade e resistividade $(\mathrm{P}<0,05)$. A insulação escrotal resultou em alterações na temperatura do escroto e na velocidade do fluxo sanguíneo testicular, porém ambos se normalizaram em um curto espaço de tempo, sugerindo uma alta eficiência dos mecanismos termorregulatórios. Portanto, os dois métodos se apresentaram eficientes para o monitoramento da dinâmica da temperatura escrotal e do fluxo sanguíneo testicular em touros submetidos à insulação escrotal. 